\title{
THE AIRCRAFT LANDING PROBLEM
}

The problem studied in the paper is an air traffic problem on the airport runway. The goal is finding an aircraft landing sequence that meets the time window for the particular aircraft and at the same time the separation times between two aircraft, which is necessary for the security of landings. The integer programming formulations and the relationship to the traveling salesman problem with cumulative costs are shown.

Keywords: mathematical programming, integer programming, scheduling

\section{The scheduling aircraft landings problem}

The problem is decision of the landing time for the set of planes, which are in the radar horizon of an air traffic controller, which involves the decision of sequencing of planes. There are two basic conditions for this time: the landing time has to lie within a specified time window and the landing times should follow a separation condition.

The lower bound of the time window for the particular plane depends on the distance of the plane from the airport and the speed of the plane, the upper bound of the time window depends on the amount of fuel. An economic speed for each plane determines the preferred landing time so called target-landing time.

The second main important constraint is the separation time between two planes. Each plane generates an air turbulence that can be dangerous for successive planes. The intensity of the turbulence depends on the type and weight of the plane. It must be specified certain time distance, separation time, between planes. There are two separation conditions:

a) complete separation conditions, if we have to ensure separation to all previous landing planes,

b) successive separation conditions, which ensure only separation to directly previous landing plane.

It can be proved that if the triangular condition for separation times is satisfied the successive separation conditions ensure the complete separation conditions. In other case the successive separation conditions are weaker than the complete separation conditions.

The goal is either a maximum number of planes scheduled in the time period or minimal mean landing time of all planes or minimal deviation of the landing times from appropriate target landing times.

The problem can be formulated for one or more runways, for landings only, or the plane take offs only or for both landings and take offs.

\section{Mathematical model of the aircraft-landing problem with completes separation.}

In [1] the following mixed integer zero-one formulation of the problem is presented.

Notation:

$P$ number of planes

$E_{i}$ the earliest landing time for plane $i(i=1,2, \ldots, P)$

$L_{i}$ the latest landing time for plane $i(i=1,2, \ldots, P)$

$T_{i}$ the target (preferred) landing time for plane $i(i=1,2, \ldots, P)$

$S_{i j}$ the required separation time between plane $i$ and plane $j$ if plane $i$ lands before plane $j$

$g_{i}>0$ the penalty cost per time unit for landing before the target time $T_{i}$ for plane $i(i=1,2, \ldots, P)$

$h_{i}>0$ the penalty cost per time unit for landing after the target time $T_{i}$ for plane $i(i=1,2, \ldots, P)$

It's supposed $E_{i} \leq T_{i} \leq L_{i}, i=1,2, \ldots, P$ and for all $i, j$.

The variables in the model are:

$t_{i}$ the landing time for the plane $i(i=1,2, \ldots, P)$

$t_{i}^{+}=\max \left[0, T_{i}-t_{i}\right]$ the landing time before target time

$t_{i}^{-}=\max \left[0, T_{i}-t_{i}\right]$ the landing time after target time

$x_{i j}=1$ if plane $i$ lands before (not necessarily directly) plane $j, i$,

$j=1,2, \ldots, P, i \neq j$

$x_{i j}=0$ otherwise.

Mathematical model of the aircraft landing problem with the complete separation:

$$
\min \sum_{i=1}^{P} g_{i} t_{i}^{+}+h_{i} t_{i}^{-}
$$

subject to

$$
\begin{aligned}
& x_{i j}+x_{j i}=1, i, j=1,2, \ldots, P, i \neq j \\
& t_{i}+S_{i j}-\left(L_{i}+S_{i j}-E_{j}\right) x_{j i} \leq t_{j}, i, j=1,2, \ldots, P, i \neq j
\end{aligned}
$$

\footnotetext{
* Jan Pelikán

University of Economics, Prague, E-mail: pelikan@vse.cz
} 


$$
\begin{aligned}
& E_{i} \leq t_{i} \leq L_{i}, i=1,2, \ldots, P \\
& t_{i}+t_{i}^{+}-t_{i}^{-}=T_{i}, i=1,2, \ldots, P \\
& t_{i}, t_{i}^{+}, t_{i}^{-} \geq 0, i=1,2, \ldots, P, x_{i j} \in\{0,1\}, \\
& i, j=1,2, \ldots, P, i \neq j
\end{aligned}
$$

The equation (2) means that either plane $i$ lands before plane $j$ or plane $j$ lands before plane $i$. The landing time $t_{j}$ should be greater than $t_{i}$ with a difference which is the separation time $S_{i j}$ (inequality (3) for $x_{j i}=0$ ). If $x_{j i}=1$, then inequality (3) is in the form $t_{i}-L_{i}+E_{j} \leq t_{j}$ and it is always satisfied due to the inequality (4).

The landing time $t_{i}$ should lie in the time window $E_{i}, L_{i}$ (the inequality (4)).

The equation (5) defines variables $t_{i}^{+}$and $t_{i}^{-}$which are the differences of $t_{i}$ from the target time $T_{i}$. The variables $t_{i}^{+}$and $t_{i}^{-}$ are not defined by (5) uniquely, nevertheless the uniqueness of $t_{i}^{+}$ and $t_{i}^{-}$is guaranteed by the fact that $g_{i}>0$ and $h_{i}>0$ in the objective function (1).

Alternative objective function is the average landing time $(1 / P) \sum_{i=1}^{P} t_{i}$. In this case the inequality (5) and the variables $t_{i}^{+}$and $t_{i}^{-}$can be dropped.

\section{Comment}

For a given sequence of planes the determination of the optimal landing times is a linear programming problem. We can obtain this model by putting all variables $x_{i j}$ to the appropriate values into the model (1)-(6).

\section{The heuristic method}

Because of NP hardness of the ALP (Aircraft Landing Problem) heuristic methods were proposed for the problem. One of them is the greedy approach [3] based on priorities numbers $p_{j}^{k}$, in which $k$-th plane is picked according to the lowest priority number $p_{j}^{k}$. The priority numbers are calculated as $p_{j}^{k}=\delta T_{j}+\epsilon E E_{j}^{k}+\alpha_{j}$, where $\delta, \epsilon$ are priority weights and $\alpha_{j}$ is a perturbation of the priority.

$E E_{j}^{k}$ is defined as the earliest time in which the plane $j$ can land given by the previous sequence of planes, that is, if the partial sequence $s_{1}, s_{2}, s_{k-1}$ of planes is constructed already, then $E E_{j}^{k}=$ $=\max \left\{E_{j}, \max _{i<k}\left[E E_{i_{i} i}+S_{s_{j}}\right]\right\}$. The next plane to land is

$$
s k=\underset{j \notin\left[s_{1}, s_{2}, \ldots, s_{k-1}\right]}{\operatorname{argmin}}, p_{j}^{k} .
$$

The earliest possible landing time for plane $s_{k}$ is $E E_{s_{k}}^{k}$.
This heuristic will not necessary find a feasible landing sequence (it is possible that $E E_{s_{k}}^{k}>L_{s_{k}}$ ), in this case we can change the parameters $\alpha_{j}$ and try it again.

\section{Mathematical model of the aircraft landing problem with successive separation.}

In this section we will solve the problem in which only successive separation is enforced. If the triangular inequality $s_{i k} \leq$ $\leq s_{i j}+s_{j k}$ for all $i \neq j \neq k$ holds, the successive separation is sufficient to ensure complete separation.

The aircraft-landing problem with successive separation can be viewed as an open traveling salesman problem with time windows, where nodes in this problem are the planes. The objective function is cumulative, so the special formulation, called traveling salesman problem with cumulative costs (or the deliveryman problem) should be used [4].

The following formulation of the aircraft-landing problem with successive separation is proposed.

Let $x_{i j}=1$ if plane $i$ lands directly before plane $j, i, j=1,2$, $\ldots, P, i \neq j, x_{i j}=0$ otherwise.

$\min \sum_{i=1}^{P} g_{i} t_{i}^{+}+h_{i} t_{i}^{-}$

$\sum_{i=1}^{P} x_{i j}=1, j=0,1, \ldots, P$

$\sum_{j=1}^{P} x_{i j}=1, i=0,1, \ldots, P$

$t_{i}+S_{i j}-\left(L_{i}+S_{i j}-E_{j}\right)\left(1-x_{i j}\right) \leq t_{j}$,

$i, j=0,1, \ldots, P, i \neq j, j>0, t_{0}=0$

$E_{i} \leq t_{i} \leq L_{i}, \quad i=1,2, \ldots, P$

$t_{i}+t_{i}^{+}-t_{i}^{-}=T_{i}, \quad i=1,2, \ldots, P$

$t_{i}, t_{i}^{+}, t_{i}^{-} \geq 0, i=1,2, \ldots, P, x_{i j} \in\{0,1\}$,

$i, j=1,2, \ldots, P, i \neq j$

The equations (8) and (9) assure that only one plane precedes and only one plane follows each plane. Plane 0 is artificial, so that $S_{o i}=S_{i 0}=0$ for all $i$.

\section{Aircraft landing problem for multiple runways}

There are two or more runways on large international airports. The plane-landing problem solves the question on which runway the plane will land and at which landing time. There are two different separation times: 
a) separation times for two planes landing on the same runway $S_{i j}$,

b) separation times for two planes landing on different runways $s_{i j}$.

It is assumed that $0 \leq s_{i j} \leq S_{i j}$.

The mathematical model (1)-(6) has to be modified so that the equation (3) for determination of landing times should be replaced by

$$
\begin{aligned}
& t_{i}+S_{i j} z_{i j}+s_{i j}\left(1-z_{i j}\right)-\left(L_{i}+S_{i j}-E_{j}\right) x_{j i} \leq t_{j}, \\
& i, j=1,2, \ldots, P, i \neq j
\end{aligned}
$$

where variable $z_{i j}$ equals 1 if planes $i$ and $j$ land on the same runway and equals 0 otherwise (the variable $z_{i j}$ does not assign the planes to runways). For $z_{i j}=1$ the constraint (14) corresponds the inequality (3), for $z_{i j}=0$ it is exchanged Sij for sij in (3).

\section{Computational results}

Many computational results with using the models shown above [1] have been published.

The problem and models are tested by the author on the data sets provided by the OR problem library maintained by Beasley (http//mscmga.ms.ic.ac.uk/info.html). There are 8 problems in this data set, all of them were solved as the same model [1] and the results were compared. The computer system for the branch and bound method LINGO ver.7 was used and run on Pentium II. The results are presented in the table 1 , where the original runtimes of the computation from [1] are written in the brackets (if they differ significantly).

\section{Acknowledgement}

The research was supported by the grant No. 402/03/1283 of the Grant Agency of the Czech Republic and research project CEZ:J18/98:311401001.
Numerical experiments

\begin{tabular}{|c|c|c|c|c|c|}
\hline $\begin{array}{l}\text { No. data } \\
\text { set }\end{array}$ & No. planes & $\begin{array}{c}\text { No. } \\
\text { Runways }\end{array}$ & \begin{tabular}{|c|} 
Cost \\
Heuristic m.
\end{tabular} & $\begin{array}{l}\text { Opt. cost } \\
\text { LINGO }\end{array}$ & $\begin{array}{c}\text { Runtime } \\
\text { (sec.) }\end{array}$ \\
\hline \multirow[t]{3}{*}{1} & 10 & 1 & 1210 & 700 & 1 \\
\hline & & 2 & 120 & 90 & 1 \\
\hline & & 3 & 0 & 0 & 1 \\
\hline \multirow[t]{3}{*}{2} & 15 & 1 & 2030 & 1480 & 6 \\
\hline & & 2 & 210 & 210 & 3 \\
\hline & & 3 & 0 & 0 & 1 \\
\hline \multirow[t]{3}{*}{3} & 20 & 1 & 2870 & 820 & 4 \\
\hline & & 2 & 60 & 60 & 2 \\
\hline & & 3 & 0 & 0 & 2 \\
\hline \multirow[t]{4}{*}{4} & 20 & 1 & 4480 & 2520 & $\begin{array}{c}548 \\
(220)\end{array}$ \\
\hline & & 2 & 680 & 640 & $\begin{array}{c}2754 \\
(1919)\end{array}$ \\
\hline & & 3 & 130 & 130 & $\begin{array}{c}75 \\
(2299)\end{array}$ \\
\hline & & 4 & 0 & 0 & 2 \\
\hline \multirow[t]{4}{*}{5} & 20 & 1 & 7120 & 3100 & $\begin{array}{l}1379 \\
(920)\end{array}$ \\
\hline & & 2 & 1220 & 650 & $(11510)$ \\
\hline & & 3 & 240 & 170 & $(1655)$ \\
\hline & & 4 & 0 & 0 & \\
\hline \multirow[t]{3}{*}{6} & 30 & 1 & 24442 & 24442 & $2(33)$ \\
\hline & & 2 & 882 & 554 & $\begin{array}{c}2482 \\
(1568)\end{array}$ \\
\hline & & 3 & 0 & 0 & 3 \\
\hline \multirow[t]{2}{*}{7} & 44 & 1 & 3974 & 1550 & $37(10)$ \\
\hline & & 2 & 0 & 0 & 5 \\
\hline \multirow[t]{3}{*}{8} & 50 & 1 & 4390 & 1950 & 77 (111) \\
\hline & & 2 & 260 & 135 & $\begin{array}{c}301 \\
(3450)\end{array}$ \\
\hline & & 3 & 0 & 0 & 9 \\
\hline
\end{tabular}

Table 1.

\section{References}

[1] BEASLEY, J. E., KRISHNAMOORTHY, M ., SHARAIHA, Y.M., ABRAMSON, D.: Scheduling aircraft landings - the static case. Transportation Science, vol. 34 No. 2 (2000)

[2] BIANCO, L., MINGOZZI, A., RICCIARDELI, S.: The Traveling Salesman Problem with Cumulative costs. Networks, vol. 23 (1993)

[3] ERNST, A. T., KRISHNAMOOTHY, M., STORER, R. H.: Heuristic and exact algorithms for scheduling aircraft landings. Networks 34 (1999)

[4] FISCHETTI, M., LAPORTE, G., MARTELLO, S.: The delivery man problem and cumulative matroids. Operations Research vol. 41, no. 6 (1993)

[5] MĚLNÍČEK, S.: Diplomová práce VŠE 2002 